\title{
Identifying and Addressing Astronomy Misconceptions in the Classroom
}

\author{
By Neil F. Comins \\ Dept. of Physics and Astronomy, Univ. of Maine, Orono, ME 04469 USA \\ GALAXY@MAINE.MAINE.EDU
}

\section{Origins of Misconceptions}

Students come into our classrooms with many misconceptions about science in general and astronomy in particular (see numerous papers and references in Novak, 1993 and Pfundt \& Duit 1993). These beliefs evolve from a variety of sources throughout childhood and adolescence (Comins, 1993a, 1993b, 1995) . I have found that directly addressing these incorrect beliefs in the context of their origins helps my students replace them with correct knowledge. By understanding the origins of their misconceptions students can screen information more effectively, i.e., they learn to think more critically. My purpose in this paper is to briefly identify origins of misconceptions and classroom techniques for replacing them.

I define misconceptions as deep seated beliefs that are inconsistent with accepted scientific information. Unless we directly address these incorrect ideas at their roots, most students cannot replace them with correct knowledge. Most students retain correct material only long enough to pass tests, and then lapse into believing their prior misconceptions.

In previous works (Comins, 1993a \& 1995) I identified a heuristic set of origins that account for all the misconceptions I have identified. It is well worth noting that such a list is by no means unique and, given that I have since added another category, nor is it complete. Nevertheless, this set of origins is an extremely practical one, providing a significant set of tools for understanding and dissecting misconceptions and how these beliefs are used by different people. In an effort to make this set more tractable, I have now revised it to an even dozen (see Sections 1.1-1.12). This was done by combining several sources, such as grouping "Over-generalization", "Belief in Object Permanence", "Belief in Object Uniqueness" and "Choosing the Simplest Explanation"together under the rubric of Common Sense. The new category is Anthropomorphism.

\subsection{Factual Misinformation}

We have all received incorrect information from sources we normally trust and, therefore, whose statements we do not question or analyze very deeply. These sources include teachers, our parents, other adults, textbooks, and knowledgeable peers.

Factual misinformation is data that has been heavily pre-processed by others before we receive it. Therefore this material often contains unstated assumptions and conditions (such as limited ranges of validity) that would affect our understanding and use of the information if we knew them. Children and others who have not yet developed techniques for critically examining information from others are especially susceptible to incorporating factual misinformation into their understanding of nature.

Some examples of misconceptions that often originate this way are that there are 12 zodiac constellations; that the North Star, Polaris, is the brightest star in the night; that the asteroids in the asteroid belt were once part of planet that was destroyed; and, that the Earth's core is liquid.

\subsection{Media Minimalism}

The news provides information that raises our interest and keeps our attention. As a result, the news about a scientific discovery often covers the most sensational, rather than the most important, elements. We incorporate the filtered, biased, incomplete, and often inaccurate information reported in the media into our view of the world and so the conclusions we draw or extrapolations we make from this knowledge are often incorrect. 


\subsection{Cartoons and Science Fiction}

Cartoons are a major external source of misconceptions about science for young children. Animators have done considerable damage to young intuitions about nature. For example, a common cartoon ploy ("The Roadrunner" comes to mind) is for the bad character to run off a cliff. When the bad guy rushes off the cliff, he stops moving forward, pauses for several seconds until the impact of what has happened is clear to both him and the young audience, and then he falls straight down.

At an adult level, people willingly suspended their disbelief in order to be entertained by exciting, but physically implausible, science fiction. Unfortunately for the general perception of nature, much science fiction actively violates physical laws. Misconceptions created by science fiction have increased dramatically in the past few decades because of the fantastic animation capabilities now available, making science fiction much more realistic than ever before. A virtually universal belief created by science fiction films is that asteroids are closely clustered together.

\subsection{Mythical Concepts}

Mythical concepts are those we accept "on faith," without sufficient evidence or without a desire to make a rational decision. Literal interpretation of the Bible, for example, requires accepting that the Earth and the rest of the universe formed in six days. Making this a foundation of one's belief about the creation of life leads to numerous misconceptions and conflicts with observations of the natural world.

Similarly, believing in magic as a supernatural power, rather than as sleight- of-hand, or in ghosts or witchcraft, requires accepting that there are rules governing the universe that are outside the realm of science. Such beliefs cause many people to interpret events in non-scientific ways that can then lead to actions contrary to those implied by scientific explanations. For example, believing that prayer can cure illnesses has led to many needless deaths and much suffering.

Related to belief in the supernatural, there is a fascinating layer of thought that everyone experiences called "magical or wishful thinking."It begins in childhood, before experience shows children how the natural world operates. Children frequently invoke magic as a means to an end they cannot otherwise achieve. By the time we are adults, such desires have evolved into "wishful thinking." At some level most of us know that wishing does not cause things to happen, but that does not stop us from trying.

\subsection{Language Imprecision}

In normal usage, most words have several meanings. We overcome imprecision in our language by taking into account the context in which words are used, as well as by adding verbiage to clarify matters or by asking questions. In science, however, every word has a single, rigorous usage. Often a scientific definition of a word is not one of the common ones in everyday usage. Among the innumerable confusing words are "black"in black hole; comet "tail"; "shooting star"; "spring"tide; and Saturn's "rings". To understand science, students must learn the correct meanings of words and not let common usages color perceptions of scientific meanings.

There are also many words that have limited meanings even to non- scientists, but most people never get the meaning correct in the first place. Common among these are "Sun" (often interpreted as a special kind of object, rather than as an ordinary star), "pulsar," "meteor", "meteoroid", and "meteorite"(the latter three often taken to have the same meaning).

\subsection{Erroneous Personal Cosmology}

Cosmological concepts and misconceptions are those that deal with the origins, sizes, locations, motions, and ages of the universe, the solar system, the stars, and the Earth. Within our "personal cosmology" we each have explanations for some, if not all, of these issues. When we encounter a question about the universe that we have never addressed explicitly, our personal cosmology is where we look first for an answer. As an example of this, ask your students where the Earth is located in the Milky Way galaxy. Referring to their personal cosmologies, students 
will often respond (incorrectly) that we are at the center of it, outside it completely, or in a spiral arm.

All of the elements of personal cosmologies lead to misconceptions. Needless to say, theology often has a bearing on these misconceptions. Notable among the non-theological beliefs are that the distances between stars and galaxies are much smaller than they actually are; that the Sun and other stars last forever; and that the Sun is immobile in space. Many of these misconceptions are based on a lack of specific information, coupled with the use of human-sized distances.

\subsection{Incomplete Understanding of the Scientific Process and Scientists}

The process of doing science includes many features that are not well understood by the public. These include expressing science in mathematical terms, making predictions from these mathematical theories, testing predictions, modifying or replacing theories that make incorrect predictions, using computers to explore the complexities of modern scientific theories, making accidental discoveries, and creating new technology to help understand nature, among others.

Most people also envision scientific information as complete, certain, and unchanging. Therefore, when they learn something about science from a "reliable source,"most people incorporate that information into their world view. Thereafter, it is very hard to change their belief about that subject. Furthermore, science is incomplete in that it does not explain everything in any discipline.

Not knowing what most scientists do, many people envision us all as white- haired men and women, spending long, lonely nights looking through telescopes in hope of making new discoveries.

\subsection{Incomplete Information/Reasoning}

Intelligent, well-educated non- scientists often reason correctly about science as far as they go, but they do not include all the scientific facts related to the subject at hand. As a result, they draw incorrect conclusions that lead to misconceptions. Furthermore, when asked about something we have never considered, we all tend to fill in the blanks in our knowledge "on the fly" and without sufficient information.

For example, a student told me he believed that the larger a star is, the more mass it must contain. His reasoning was that the greater the volume, the greater the mass packed into it. What he did not realize is that densities vary from star to star.

\subsection{Misinterpreting Sensory Information}

Our senses pre-filter incoming information before it enters our brains. For example, our eyes are not uniformly sensitive to all the colors of the rainbow, being most responsive to yellow light. Sensitivity to color falls off towards both red and violet. Therefore, what we perceive as the color of each object is not its true color, because the intensities of all the colors from it are changed in our eyes before the color information enters our brain. The same applies to the other senses, as people who differentially lose sensitivity to pitch know all too well.

Furthermore, even when we see a true representation of events, our uncritical interpretations of them are often incorrect (see also Common Sense, below). Examples of misconstrued perceptions include mirages, observations that the Sun, planets, and stars orbit the Earth (they certainly appear to do so); that the Earth is flat; that the Sun is yellow; and that stars twinkle.

\subsection{Inaccurate or Incomplete Observations}

People observe things inaccurately for a variety of reasons. These include not knowing what to look for (a person believing that all the stars in the sky are white is ill-prepared to notice that many of them are colored); not being sufficiently interested in making careful observations; being distracted by certain features of what they see; mis- categorizing what they see; and being biased by what they have been told beforehand.

Other examples are that the same constellations are seen at night throughout the year, that 
no planets are visible with the naked eye, that the Moon is only visible at night, and that the sword of Orion is just composed of stars.

\subsection{Anthropomorphizing}

Among the myriad things every child must learn is that they are separate entities from their care givers and from the rest of the world. This realization takes several years and the earlier stage of seamless attachment children have to parents and other care givers leaves traces in the anthropomorphizing that virtually all of us do as adults. We attribute human motives and desires to creatures who function solely by instinct and autonomic response to the outside world, as well as to plants and trees, and to inanimate objects like the celestial bodies. By responding to the rest of the natural world as if it had awareness and other human traits, we develop misunderstandings about how things work.

\subsection{Common Sense}

Common sense, defined as "sound practical judgement that is independent of specialized knowledge or training" (Webster's College Dictionary), is an essential facet of our interaction with the world. While it is often useful in everyday life, common sense frequently leads us to believe concepts inconsistent with the laws and behavior of nature. The problem is that so much of what goes on in the natural world defies "common sense."For example, common sense tells us that heavier objects will drop faster than lighter ones. The difference between common sense and scientific fact has led to a growing divergence between what most people believe and what scientists know to be correct.

Common sense overlaps a variety of other sources of misconceptions. Also, the "common sense" activities we each use in reconciling new information with current beliefs depend on learning patterns we developed as children. Here are some specific error-prone common sense activities:

\subsubsection{Over-generalization}

The invalid generalization from the properties of objects we know to ones we do not know causes many misconceptions. We make these generalizations both as we are learning new things and as we are asked about things we have never thought about before. For example, when asked to describe the surfaces of the other planets, most people generalize, from the Earth, that they have solid surfaces. When asked to describe the other moons in the solar system, many people say that they are all spherical, like our Moon.

\subsubsection{Uniqueness}

Incorrectly assuming that objects are unique has the opposite effect to over-generalizing. For example, because Earth is the only body in the solar system with life on it, many people believe that the Earth is also the only body with water. Many people also believe that Earth is the only planet with an atmosphere.

\subsubsection{Permanence}

Most children develop a belief that their social world is static, meaning that the people and objects dear to them will be around forever. This belief is motivated by the fear of change (desire for permanence), a feeling that often continues into adulthood and is therefore applied to new situations and concepts by adults. A belief in permanence can also originate in the lack of perceived change in the Earth or its cycles. In most places on Earth the planet's surface does not change during our lifetimes; the day is always twenty-four hours long; and, the same patterns of stars are visible each year. We know, of course, that while these cycles are reliable over the span of a human life, belief in permanence on astronomical time scales is completely unfounded. 


\subsubsection{Choosing the Simplest Explanation}

Most people choose the first, and therefore usually the simplest, explanation that they think of for a new situation. Referring to that most ubiquitous of misconceptions, most people assert that summers are hotter than winters by analogy with the fact that the closer you are to a fire, the warmer you feel.

\section{Classroom Remediation of Misconceptions}

Remediating misconceptions is made difficult by several factors. First, of course, people are resistant to the idea that their beliefs are incorrect. As a result, we tend to take new information and distort and modify it to make it consistent with our current understanding of any topic. Second, many misconceptions are used to help explain a variety of other things, so removing a misconception can undermine many other ideas we hold. Third, different people give different weight to different common sense beliefs, such as the uniqueness of objects and ideas, or the power of generalization. As a result, different people often hold diametrically opposite misconceptions on a given topic. For example, those people who focus on uniqueness often assert that our Moon is the only moon in the solar system, while those who over-generalize often believe that each planet has one moon.

\subsection{Make students aware that they harbor misconceptions}

This is hard because nobody likes to be told that their beliefs are wrong. The most effective ways I have found of doing this are by using counterintuitive physics demonstrations (levitating a beach ball in a tilted air stream works well) and by taking on some common astronomical misconceptions at the beginning of the semester. That changing distance from the Sun does not cause the seasons and that the Sun is not yellow (i.e., that its most intense radiation is not yellow, but blue- green) are useful.

The process of awakening misconception awareness is important. Invite students to think about (and if they are brave or confident enough, tell) what they believe to be correct before doing a demonstration or giving a correct answer.

"How many of you think that the seasons are caused by the changing distance from the Earth to the Sun?" This type of question is valuable because it makes the students active participants in the process. It also invariably creates anxiety, which should also be acknowledged and explained to the students as useful in confronting incorrect beliefs.

Less effective are questions like "What causes the seasons?" "What color is the Sun?" "What is the shape of the Earth's orbit around the Sun?" "What planet is farthest from the Sun?"The problem with this format of questioning is that if a student confidently gives the correct answer, it can dissuade other students with incorrect beliefs from getting involved.

\subsection{Assure students that misconceptions are unavoidable and that they do not imply stupidity}

Corollary: Students (indeed, everyone) look for someone to blame for their own misinformation. In this case, that is often justified. Anger and frustration often focuses on teachers, religious leaders, parents, friends, the media.

\subsection{Teach students the origins of misconceptions}

The importance of knowing where misconceptions come from is that it helps students more readily accept that they have received or processed misinformation in the past and it helps them evaluate their sources of information more critically in the future. Changing ones' beliefs in the face of irrefutable evidence is hard enough; doing so without knowing how and where the incorrect information that we have originally came from is much harder. Knowing, for example, that our senses are not "ideal" makes us more sensitive to other possible interpretations of what we perceive. 


\subsection{Assure students that with suitable effort, they can unlearn many misconceptions}

As discussed above, the process is not easy, but misconceptions can be replaced with correct knowledge. I have found that the more students understand the process of replacing incorrect information, the more apt they are to try and change.

\subsection{Teach the scientific method and critical thinking}

As noted above, most students do not understand what science is, how it functions, or how scientists operate (see, e.g., Lett, 1990). It is worth introducing students to the relationship between theories and observations/experiments, as well as to the predictive power, falsifiability, repeatability, and simplicity (i.e., Occam's razor) of scientific theories.

\subsection{Ask "What If?"questions about misconception-prone topics and explore the results}

"What if?"questions allow you to show students the implications of both incorrect and correct beliefs (Comins, 1993c). By following misconceptions to their logical contradictions, you can forcefully demonstrate why some incorrect ideas must be changed. An example of this is assuming that the Sun is shining by burning gases. Assuming that the Sun's entire mass was composed of fuel and oxidizer, it would have completed combustion billions of years ago and "gone out."In that case, of course, we could not be here.

\subsection{Keep students focused on this issue throughout the course}

Point out common misconceptions throughout lectures. I often offer extra credit to students who provide me with lists of misconceptions (along with a statement of the origins of each belief) that $I$ corrected for them during the course. This incentive keeps them thinking about their prior beliefs, the origins of these beliefs, and the difference between what I say and what they thought they knew. I also include pedagogy designed to keep students focused on misconceptions throughout my new textbook (Kaufmann and Comins, 1996).

\section{REFERENCES}

Comins, N.F., Misconceptions About Astronomy: Their Origins, 1993a, in, Proc. of the 3rd Inter. Sem. on Misconceptions in Sci. and Math. ed Novak, J.D. (see below).

Comins, N.F., Misconceptions About Astronomy: Their Origins and Effects on Teaching, 1993b, abstract in Bulletin of the Amer. Astro. Society, 25, \#4, 1430.

Comins, N.F.,What If the Moon Didn'T Exist? 1993c, New York: HarperCollins.

Comins, N.F., Addressing Common Astronomy Misconceptions in the Classroom, 1995, in Astro. Soc. of the Pacific Conf. 89, "Astronomy Education," ed. John Percy.

Kaufmann, W.J.III, \& N.F. Comins,1996, Discovering the Universe 4th, NY:Freeman.

LETT, J., A Field Guide to Critical Thinking, in Skeptical Inquirer, V14, Wint.1990.

NovaK, J.D., 1993, Proceedings of the Third International Seminar on Misconceptions and Educational Strategies in Science and Math, Cornell University, Homepage: http://meaningful.education.cornell.edu/miscon/homepage.htm

Pfundt, H. \& Reinders D., 1993, Bibliography: Students' Alternative Frameworks and Science Education, Kiel, Germany: IPN at the Univ. of Kiel. 